\title{
Polymer Nanocontainers
}

\author{
Wolfgang Peter Meier* \\ Ruzicka Prize Winner 2001
}

\begin{abstract}
In recent years nanostructured materials have attracted increasing economical and scientific interest. The idea is to create new properties and new applications with well-known standard materials by controlling their size and morphology at the nanometer-size level. In this context polymer nanocontainers have proven to be particularly interesting. The preparation of nanometer-sized containers that are both biocompatible and stable is, however, a very challenging task. The most promising materials in this respect are amphiphilic block copolymers that can be easily designed to mimic biological membranes. Similar to conventional lipids, appropriate amphiphilic block copolymers may also self-assemble in aqueous media to vesicular structures that can be stabilized by polymerization. The long-term stability of these structures makes them well adapted for applications and guarantees a constant nonchanging environment for embedded therapeutic or analytic molecules and promise interesting applications in pharmacy or biotechnology. Particularly since the permeability of such nanocontainers can be controlled by attaching stimuli-sensitive (i.e. pH, temperature, specific molecules,...) groups to their surface or by inserting specific channel proteins into their shells. Furthermore, surface-bound ligands or reconstituted membrane proteins makes them suitable for molecular recognition.
\end{abstract}

Keywords: Amphiphilic block copolymers · Membrane proteins · Polymer nanostructures · Self assembly

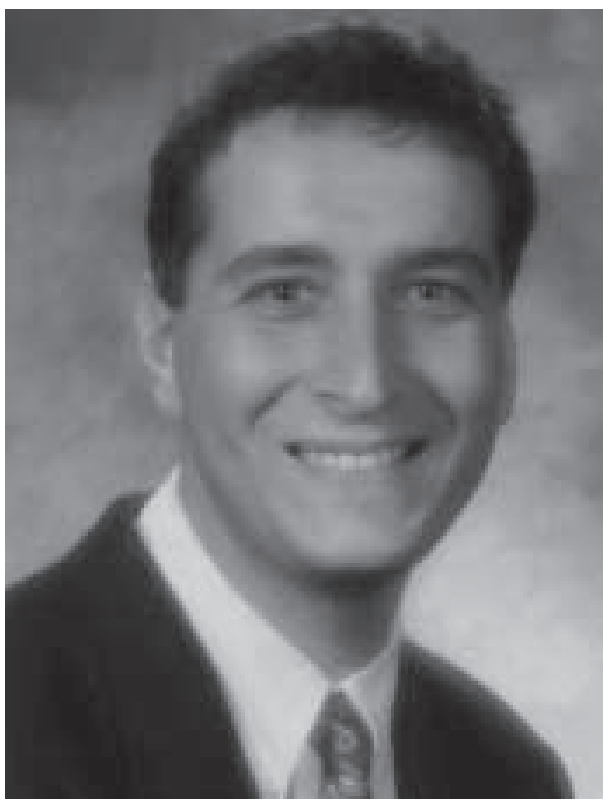

Wolfgang Peter Meier was born on September 11, 1964 in Biberach (Germany). From 1984 to 1989 he studied chemistry at the University of Freiburg (Germany). He completed his diploma thesis in polymer chemistry under the supervision of Prof. Dr. H. Finkelmann: 'Synthesis and Characterization of New Cholesteric Side-chain Liquid Crystalline Polymers'. From 1989 to 1992 he worked for his $\mathrm{PhD}$ in polymer chemistry, supervisor Prof. Dr. H. Finkelmann, on 'Piezoelectricity of Cholesteric Elastomers'. After his $\mathrm{PhD}$, he undertook postdoctoral research (1993-1996) in the group of Prof. Dr. H.-F. Eicke at the Institute of Physical Chemistry of the University of Basel (Switzerland). From October 1993 he was the project manager of Swiss National Science Foundation research projects, and from 1996 a lecturer for physical chemistry at the University of Basel and leader of an independent research group. In 1998 he completed his habilitation thesis. In September 2001he became a professor at the International University of Bremen. 